\title{
Cyprinid phylogeny based on Bayesian and maximum likelihood analyses of partitioned data: implications for Cyprinidae systematics
}

\author{
WANG XuZhen ${ }^{1}$, GAN XiaoNi ${ }^{1}$, LI JunBing ${ }^{1}$, MAYDEN Richard L. ${ }^{2}$ \& HE ShunPing ${ }^{1 *}$ \\ ${ }^{1}$ Key Laboratory of Aquatic Biodiversity and Conservation of Chinese Academy of Sciences, Institute of Hydrobiology, Chinese Academy of \\ Sciences, Wuhan 430072, China; \\ ${ }^{2}$ Department of Biology, Saint Louis University, Saint Louis, Missouri 63103-2010, USA
}

Received April 25, 2012; accepted July 16, 2012

\begin{abstract}
Cyprinidae is the biggest family of freshwater fish, but the phylogenetic relationships among its higher-level taxa are not yet fully resolved. In this study, we used the nuclear recombination activating gene 2 and the mitochondrial 16S ribosomal RNA and cytochrome b genes to reconstruct cyprinid phylogeny. Our aims were to (i) demonstrate the effects of partitioned phylogenetic analyses on phylogeny reconstruction of cyprinid fishes; (ii) provide new insights into the phylogeny of cyprinids. Our study indicated that unpartitioned strategy was optimal for our analyses; partitioned analyses did not provide better-resolved or -supported estimates of cyprinid phylogeny. Bayesian analyses support the following relationships among the major monophyletic groups within Cyprinidae: (Cyprininae, Labeoninae), ((Acheilognathinae, ((Leuciscinae, Tincinae), Gobioninae)), Xenocyprininae). The placement of Danioninae was poorly resolved. Estimates of divergence dates within the family showed that radiation of the major cyprinid groups occurred during the Late Oligocene through the Late Miocene. Our phylogenetic analyses improved our understanding of the evolutionary history of this important fish family.
\end{abstract}

Cyprinidae, phylogeny, molecular dating, partitioned analyses

Citation: Wang X Z, Gan X N, Li J B, et al. Cyprinid phylogeny based on Bayesian and maximum likelihood analyses of partitioned data: implications for Cyprinidae systematics. Sci China Life Sci, 2012, 55: 761-773, doi: 10.1007/s11427-012-4366-Z

The family Cyprinidae is the largest freshwater fish family and includes an estimated 2420 species in about 220 genera [1]. The large number of species, wide geographic distribution, and considerable morphological diversity make the cyprinid fishes taxonomically difficult [2] and a challenge for cladistic analysis. The history of Cyprinidae classification was well documented by Hensel [3], and numerous efforts have been made to partition cyprinids into subfamilies using morphological or anatomical characteristics [2,4-8]. However, the systematic relationships among many cyprinid subfamilies are poorly understood, because the

*Corresponding author (email: clad@ihb.ac.cn) subfamilies are vaguely defined or supported by few morphological characteristics [2].

Cyprinidae has been conventionally divided into two major lineages, the cyprinine (barbine) and the leuciscine groups. Overall, morphology has provided few insights into cyprinid relationships below the family level and failed to reach agreement on the number and the monophyly of subfamilies within Cyprinidae. Chen et al. [8] published the cladistic evaluation of cyprinid subfamily relationships and the additional morphological studies by Cavender and Coburn [9] and Howes [2] attempted a coherent classification of all cyprinid groups, including the monotypic genus Tinca. In these previous studies, conflicting arrangements of the 
subfamilies Tincinae, Rasborinae, and Gobioninae were proposed.

Recently, molecular phylogenetic analyses have been performed on Cyprinidae. In general, most of the molecular studies of European cyprinids [10-16] have been phylogenetically congruent. For example, all of these studies supported the nesting of Alburninae [2] within a paraphyletic Leuciscinae, but not the usual dichotomy between barbelled cyprinines (subfamilies Cyprininae, Gobioninae, and Rasborinae) and leuciscines lacking or sporadically possessing barbels (subfamilies Acheilognathinae, Leuciscinae, Cultrinae, and Alburninae) [2]. Because cyprinids are most diverse in Asiatic waters [17], phylogenetic studies that include Asian species would greatly advance cyprinid systematics [18-20]. Cunha et al. [18] identified an Asian group consisting of cultrins+acheilognathins+gobionins+ xenocyprinins within the Cyprinidae using cytochrome $b$ (Cytb) gene sequences. Other molecular phylogenies of East Asian cyprinids indicated two principal lineages within $\mathrm{Cy}-$ prinidae and provided phylogenetic evidence for the monophyly of cultrins-xenocyprins and affiliated groups $[19,20]$. However, these molecular analyses were heavily based on partial mtDNA sequences, and resulted in phylogenetic trees with limited resolution and little discrimination among alternative phylogenetic hypotheses.

The current cyprinid classification developed in the absence of a strong phylogenetic framework and is largely morphology based; few revisions have resulted from recent molecular evidence, due to the limited taxon sampling in those studies. Some critical areas of Cyprinidae phylogeny and systematics remain unresolved. First, a majority of designated cyprinid subfamilies have not been tested for monophyly with either molecular or morphological data, and molecular data [18-20] has failed to support the monophyly of many morphologically-defined subfamilies, e.g., Rasborinae [8] and Leuciscinae [2,4]. Second, previous analyses have not agreed on the phylogenetic positions of Rasborinae, Tincinae, and Acheilognathinae. In recent molecular phylogenies, relationships among these subgroups remained unclear, because corresponding nodes were generally not statistically supported. Third, although the leuciscine and cyprinine subdivisions of Cyprinidae are widely accepted, the higher-level taxonomic relationships within these clades remain unresolved.

Molecular phylogenetic analyses of East Asian cyprinid resulted in substantial disagreement on the classification of subfamilies compared with the traditional taxonomy $[19,20]$. Therefore, extensive sampling of Asian cyprinids would provide further insights into the phylogenetic systematics of this family. The present paper used extensive taxon sampling and concatenated sequence data for the nuclear recombination activating gene 2 (RAG2) and the mitochondrial 16S ribosomal RNA (16S rRNA) and Cytb genes to reconstruct the phylogeny of cyprinids.

To analyze DNA sequence data-sets with multiple genes, partitioned phylogenetic analyses have become increasingly popular in recent years. Partitioned phylogenetic analyses use separate nucleotide substitution models (and associated parameters) for subsets of the data, to better explore partition-specific models of evolution and to reduce systematic error, thus yielding more accurate phylogenies. Generally, partitioned phylogenetic analyses are undertaken in a Bayesian framework [21], but recently, mixed-model search methods using maximum likelihood (ML) have become available [22]. Furthermore, an appropriately-partitioned data-set should be well modeled but not over-partitioned, because the over-parameterization (including over-partitioning) could result in parameter nonidentifiability, increased variance, improper posterior distributions, and undue influence of the priors [23]. Alternatives to Bayes factors for phylogenetic model selection that use explicit parameterization penalties are now available for partitioned analyses [23].

We performed ML and Bayesian analyses of partitioned data to reconstruct the phylogeny of cyprinid fishes, and also used relaxed molecular clock approaches to estimate the dates of cladogenetic events within the family. Our main aims were (i) to demonstrate how partitioning concatenated data affected phylogenetic reconstruction of cyprinid fishes; (ii) to test the monophyly of the currently-recognized subfamilies within Cyprinidae; and (iii) to discuss the taxoonomic implications of the recovered clades.

\section{Materials and methods}

\subsection{Taxon sampling and total DNA isolation}

Our samples include 103 cyprinid species representing all major morphological groups and all 12 subfamilies within Cyprinidae [4]. Outgroup taxa were selected based on the consensus that Cypriniformes is a monophyletic group [24,25]. Therefore, six cypriniform fishes outside Cyprinidae were included in our analyses (Catostomidae, Balitoridae, Cobitidae, and Gyrinocheilidae) (Table 1).

Field-collected fish muscle or fin tissues were fixed in $95 \%$ ethanol and kept at $-20^{\circ} \mathrm{C}$ in the laboratory until DNA extraction. Total genomic DNA was isolated from muscle or fin tissues using the phenol/chloroform extraction procedure [26].

\subsection{DNA sequences collection and alignment}

The nuclear RAG2 gene and mitochondrial genes were amplified from total DNA extracts via polymerase chain reaction (PCR) using published and/or optimized primers [27-29]. Reaction mixtures contained approximately $100 \mathrm{ng}$ of DNA template, $5 \mu \mathrm{L}$ of $10 \times$ reaction buffer, $2 \mu \mathrm{L}$ dNTPs (each $2.5 \mathrm{mmol} \mathrm{L}^{-1}$ ), 2.0 U Taq polymerase, and $1 \mu \mathrm{L}$ of each oligonucleotide primer $\left(10 \mu \mathrm{mol} \mathrm{L} \mathrm{L}^{-1}\right.$ each $)$, in a final volume $50 \mu \mathrm{L}$. The PCR amplification profile included an 
Table 1 Cyprinid ingroup and cypriniform outgroup taxa used in this study and GenBank accession numbers of the sequence data ${ }^{\text {a) }}$

\begin{tabular}{|c|c|c|c|c|c|}
\hline \multirow{2}{*}{ Subfamily } & \multirow{2}{*}{ Taxa } & \multirow{2}{*}{ Sampling location } & \multicolumn{3}{|c|}{ Accession No. } \\
\hline & & & RAG2 & 16S rRNA & Cytb \\
\hline \multirow[t]{24}{*}{ Barbinae } & Acrossocheilus beijiangensis & Rong'an, Guangxi Zhuang Auto. Region & DQ366967 & DQ845869 & - \\
\hline & Acrossocheilus elongates & Rong'an, Guangxi Zhuang Auto. Region & DQ366979 & GQ406254 & - \\
\hline & Acrossocheilus hemispinus & Rong'an, Guangxi Zhuang Auto. Region & DQ366986 & DQ845867 & GQ406312 \\
\hline & Balantiocheilos melanopterus & Aquarium, Wuhan & DQ366933 & GQ406255 & - \\
\hline & Barbodes huangchuchieni & Mengla, Yunnan Prov. & DQ366952 & GQ406256 & - \\
\hline & Barbodes vernayi & Mengla, Yunnan Prov. & DQ366987 & DQ845870 & GQ406313 \\
\hline & Barbonymus schwanenfeldii & Aquarium, Wuhan & DQ366961 & DQ845906 & AF180823* \\
\hline & Barbus barbus & France & DQ366990 & DQ845879 & $\mathrm{AB} 238965^{*}$ \\
\hline & Barbus sp. & Africa & DQ366980 & DQ845860 & AF180842* \\
\hline & Hampala macrolepidota & Mengla, Yunnan Prov. & DQ366965 & DQ845863 & DQ464974* \\
\hline & Onychostoma gerlachi & Jinghong, Yunnan Prov. & DQ366963 & DQ845862 & GQ406314 \\
\hline & Onychostoma leptura & Xilin, Guangxi Zhuang Auto. Region & DQ366955 & GQ406257 & - \\
\hline & Onychostoma macrolepis & Taian, Shandong Prov. & DQ366942 & GQ406258 & - \\
\hline & Onychostoma sima & Hejiang, Sichuan Prov. & DQ366991 & DQ845861 & - \\
\hline & Percocypris pingi pingi & Hejiang, Sichuan Prov. & DQ366962 & GQ406259 & - \\
\hline & Puntius semifasciolatus & Jinghong, Yunnan Prov. & DQ366951 & GQ406260 & AY856116* \\
\hline & Puntius conchonius & Aquarium & GQ406253 & DQ845880 & AY004751* \\
\hline & Puntius tetrazona varieties & Aquarium & DQ366938 & EU287909 & EU287909* \\
\hline & Sikukia stejnegeri & Mengla, Yunnan Prov. & DQ366931 & DQ845872 & GQ406315 \\
\hline & Sinocyclocheilus tingi & Fuxian Lake, Yunnan Prov. & DQ366978 & DQ845866 & AY854701* \\
\hline & Spinibarbus hollandi & Tunxi, Anhui Prov. & DQ366973 & DQ845865 & AY195629* \\
\hline & Tor douronensis & Menglun, Yunnan Prov. & DQ366945 & DQ845877 & DQ464986* \\
\hline & Tor qiaojiensis & Yingjiang, Yunnan Prov. & DQ366970 & DQ845873 & GQ406316 \\
\hline & Tor sinensis & Mengla, Yunnan Prov. & DQ366936 & DQ845876 & FJ211164* \\
\hline \multirow[t]{4}{*}{ Cyprininae } & Carassius auratus & Wuhan, Hubei Prov. & DQ366941 & $\mathrm{AB} 006953$ & AB006953* \\
\hline & Cyprinus carpio & Tian'e, Guangxi Zhuang Auto. Region & DQ366994 & $\mathrm{X} 61010^{*}$ & $\mathrm{X} 61010^{*}$ \\
\hline & Cyprinus multitaeniata & Guiping, Guangxi Zhuang Auto. Region & DQ366939 & DQ845845 & - \\
\hline & Procypris rabaudi & Hejiang, Sichuan Prov. & DQ366969 & DQ845846 & GQ406317 \\
\hline \multirow[t]{20}{*}{ Labeoninae } & Cirrhinus molitorella & Tengxian, Guangxi Zhuang Auto. Region & DQ366959 & DQ845883 & AY463098* \\
\hline & Crossocheilus latius & Tengchong, Yunan Prov. & DQ366982 & DQ845882 & - \\
\hline & Crossocheilus reticulates & Menglun, Yunnan Prov. & DQ366937 & GQ406261 & - \\
\hline & Discogobio bismargaritus & Liuzhou, Guangxi Zhuang Auto. Region & DQ366947 & DQ845890 & GQ406318 \\
\hline & Discogobio brachyphysallidos & Jinxiu, Guangxi Zhuang Auto. Region & DQ366958 & DQ845901 & GQ406319 \\
\hline & Discogobio laticeps & Tian'e, Guangxi Zhuang Auto. Region & DQ366949 & DQ845889 & GQ406320 \\
\hline & Epalzeorhynchos frenatus rar & Aquarium, Jinghong & DQ366943 & DQ845905 & GQ406321 \\
\hline & Garra kempi & Chayu, Tibet Auto. Region & DQ366968 & DQ845885 & - \\
\hline & Garra orientalis & Ledong, Hainan Prov. & DQ366957 & DQ845884 & GQ406322 \\
\hline & Garra taeniata & Jinghong, Yunnan Prov. & DQ366953 & GQ406262 & - \\
\hline & Henicorhynchus lineatus & Menglun, Yunnan Prov. & DQ366935 & GQ406263 & GQ406323 \\
\hline & Labeo yunnanensis & Mengla, Yunnan Prov. & DQ366948 & DQ845881 & GQ406324 \\
\hline & Lobocheilus melanotaenia & Menglun, Yunnan Prov. & DQ366940 & DQ845902 & DQ464990* \\
\hline & Osteochilus salsburyi & Rong'an, Guangxi Zhuang Auto. Region & DQ366971 & DQ845892 & GQ406325 \\
\hline & Parasinilabeo assimilis & Rong'an, Guangxi Zhuang Auto. Region & DQ366992 & DQ845887 & GQ406326 \\
\hline & Pseudocrossocheilus bamaensis & Tian'e, Guangxi Zhuang Auto. Region & DQ366993 & DQ845895 & GQ406327 \\
\hline & Ptychidio jordani & Tian'e, Guangxi Zhuang Auto. Region & DQ366974 & DQ845893 & GQ406328 \\
\hline & Rectoris posehensis & Dou'an, Guangxi Zhuang Auto. Region & DQ366975 & DQ845891 & GQ406329 \\
\hline & Semilabeo notabilis & Jinxiu, Guangxi Zhuang Auto. Region & DQ366983 & DQ845886 & GQ406330 \\
\hline & Sinilabeo rendahli & Yidu, Hubei Prov. & DQ366932 & GQ406264 & - \\
\hline \multirow[t]{6}{*}{ Schizothoracinae } & Gymnocypris eckloni eckloni & Huanghe, Qinghai Prov. & DQ366950 & DQ845853 & AY463522* \\
\hline & G. przewalskii przewalskii & Qinghai Lake, Qinghai Prov. & DQ366954 & DQ845851 & AY463523* \\
\hline & Gymnodiptychus dybowskii & Yili, Xinjiang Uygur Auto. Region & DQ366956 & DQ845859 & AY463513* \\
\hline & $\begin{array}{l}\text { Schizopygopsis younghusbandi } \\
\text { younghusbandi }\end{array}$ & Bomi, Tibet Auto. Region & DQ366976 & GQ406265 & AY463501* \\
\hline & Schizothorax dulongensis & Guyong, Yunnan Prov. & DQ366985 & DQ845849 & AY954284* \\
\hline & Schizothorax meridionalis & Yingjiang, Yunnan Prov. & DQ366989 & DQ845847 & AY954287* \\
\hline
\end{tabular}


(Continued)

\begin{tabular}{|c|c|c|c|c|c|}
\hline \multirow{2}{*}{ Subfamily } & \multirow{2}{*}{ Taxa } & \multirow{2}{*}{ Sampling location } & \multicolumn{3}{|c|}{ Accession No. } \\
\hline & & & RAG2 & 16S rRNA & Cytb \\
\hline \multirow[t]{3}{*}{ Schizothoracinae } & Schizothorax molesworthi & Chayu, Tibet Auto. Region & DQ366946 & DQ845848 & DQ126130* \\
\hline & Schizothorax myzostomus & Guyong, Yunnan Prov. & DQ366960 & DQ845850 & GQ406331 \\
\hline & Schizothorax waltoni & Chayu, Tibet Auto. Region & DQ366981 & GQ406266 & AY463518* \\
\hline \multirow[t]{14}{*}{ Leuciscinae } & Cyprinella lutrensis & GN531 & DQ367019 & GQ406267 & $\mathrm{AB} 070206^{*}$ \\
\hline & Leuciscus leuciscus & France & DQ367007 & GQ406268 & AY509823* \\
\hline & Phoxinus phoxinus & Europe & DQ367022 & GQ406269 & Y10448* \\
\hline & Phoxinus lagowskii & Hengren, Liaoning Prov. & DQ367035 & GQ406270 & $\mathrm{AB} 162650^{*}$ \\
\hline & Rutilus rutilus & France & DQ367003 & GQ406271 & AF095610* \\
\hline & Pimephales promelas & GN530 & DQ367000 & GQ406272 & AF117203* \\
\hline & Rhinichthys atratulus & GN529 & DQ367018 & GQ406273 & AF452078* \\
\hline & Elopichthys bambusa & Taoyuan, Hunan Prov. & DQ367016 & GQ406274 & GQ406332 \\
\hline & Ochetobius elongates & Taoyuan, Hunan Prov. & DQ367012 & GQ406275 & AF309506 \\
\hline & Luciobrama macrocephalus & Tengxian, Guangxi Zhuang Auto. Region & DQ367013 & GQ406276 & - \\
\hline & Ctenopharyngodon idella & Hengxian, Guangxi Zhuang Auto. Region & DQ366996 & GQ406277 & AF051860* \\
\hline & Mylopharyngodon piceus & Taoyuan, Hunan Prov. & DQ367011 & GQ406278 & AF051870* \\
\hline & Squaliobarbus curriculus & Wuhan, Hubei Prov & DQ367021 & GQ406279 & $\mathrm{AF} 051877^{*}$ \\
\hline & Tinca tinca & Europe & DQ367029 & GQ406280 & Y10451* \\
\hline \multirow[t]{2}{*}{$\begin{array}{l}\text { Hypophthalmich- } \\
\text { thyinae }\end{array}$} & Aristichthys nobilis & Wuhan, Hubei Prov. & DQ367038 & GQ406281 & $\mathrm{AF} 051855^{*}$ \\
\hline & Hypophthalmichthys molitrix & Chenxi, Hunan Prov. & DQ367002 & GQ406282 & AF051866 \\
\hline \multirow[t]{3}{*}{ Xenocyprinae } & Distoechodon tumirostris & Wuhan, Hubei Prov. & DQ366998 & GQ406283 & AF336308* \\
\hline & Pseudobrama simony & Taoyuan, Hunan Prov. & DQ367028 & GQ406284 & AF036194* \\
\hline & Xenocypris argentea & Taoyuan, Hunan Prov. & DQ367024 & GQ406285 & AP009059* \\
\hline \multirow[t]{8}{*}{ Danioninae } & Danio apogon & Mengla, Yunnan Prov. & DQ367039 & GQ406286 & - \\
\hline & Danio rerio & & U71094* & $\mathrm{AC} 024175^{*}$ & $\mathrm{AC} 024175^{*}$ \\
\hline & Hemigrammocypris rasborella & Japan & DQ367008 & GQ406287 & AF375863* \\
\hline & Nicholsicypris normalis & Diaoluoshan, Hainan Prov. & DQ367034 & GQ406288 & - \\
\hline & Opsariichthys bidens & Taoyuan, Hunan Prov. & DQ367014 & GQ406289 & DQ367044* \\
\hline & Raiamas guttatus & Mengla, Yunnan Prov. & DQ366966 & GQ406290 & $\mathrm{AF} 051875^{*}$ \\
\hline & Tanichthys albonubes & Aquarium, Wuhan & DQ367023 & GQ406291 & $\mathrm{EF} 151121^{*}$ \\
\hline & Zacco platypus & Jinxiu, Guangxi Zhuang Auto. Region & DQ367010 & GQ406292 & AY245048* \\
\hline \multirow[t]{8}{*}{ Cultrinae } & Culter alburnus & Taoyuan, Hunan Prov. & DQ367004 & GQ406293 & AP009060* \\
\hline & Cultrichthys erythropterus & Lingshan, Guangxi Zhuang Auto. Region & DQ367037 & GQ406299 & $\mathrm{AF} 051859^{*}$ \\
\hline & Megalobrama amblycephala & Wuhan, Hubei Prov. & DQ367025 & GQ406294 & AF051867* \\
\hline & Pseudohemiculter dispar & Rong'an, Guangxi Zhuang Auto. Region & DQ367001 & GQ406296 & - \\
\hline & Pseudolaubuca sinensis & Taoyuan, Hunan Prov. & DQ367017 & GQ406297 & - \\
\hline & Rasborinus lineatus & Hengxian, Guangxi Zhuang Auto. Region & DQ367036 & GQ406298 & - \\
\hline & Sinibrama macrops & Rong'an, Guangxi Zhuang Auto. Region & DQ367006 & GQ406295 & - \\
\hline & Toxabramis swinhonis & Bobai, Guangxi Zhuang Auto. Region & DQ367027 & GQ406300 & DQ464972* \\
\hline \multirow[t]{2}{*}{ Gobiobotinae } & Gobiobotia abbreviate & Tian'e, Guangxi Zhuang Auto. Region & DQ367033 & GQ406301 & - \\
\hline & Gobiobotia filifer & Wuhan, Hubei Prov. & DQ367032 & GQ406302 & AY953002* \\
\hline \multirow[t]{7}{*}{ Gobioninae } & Abbottina rivularis & Nanchong, Sichuan Prov. & DQ366995 & GQ406303 & $\mathrm{AF} 051856^{*}$ \\
\hline & Coreius heterodon & Wuhan, Hubei Prov. & DQ367005 & GQ406304 & AY $953000^{*}$ \\
\hline & Gobio gobio & France & DQ367015 & GQ406305 & AY426592* \\
\hline & Pseudogobio vaillanti & Tian'e, Guangxi Zhuang Auto. Region & DQ366999 & GQ406306 & AY953019* \\
\hline & Pseudorasbora parva & Jinxiu, Guangxi Zhuang Auto. Region & DQ366997 & GQ406307 & $\mathrm{AF} 051873^{*}$ \\
\hline & Sarcocheilichthys sinensis sinensis & Hejiang, Sichuan Prov. & DQ367026 & GQ406308 & AY952983* \\
\hline & Saurogobio dabryi & Changyang, Hubei Prov. & DQ367020 & GQ406309 & AY245091* \\
\hline \multirow[t]{2}{*}{ Acheilognathinae } & Paracheilognathus meridianus & Hengxian, Guangxi Zhuang Auto. Region & DQ367009 & GQ406310 & - \\
\hline & Rhodeus sp & Xilin, Guangxi Zhuang Auto. Region & DQ367031 & GQ406311 & DQ026430* \\
\hline \multirow[t]{6}{*}{ Outgroup } & Micronemacheilus pulcher & Rong'an, Gaungxi Zhuang Auto. Region & DQ367041 & DQ845921 & DQ105199* \\
\hline & Myxocyprinus asiaticus & Wuhan, Hubei Prov. & DQ367043 & DQ845896 & AY $986503^{*}$ \\
\hline & Paramisgurnus dabryanus & Rong'an, Guangxi Zhuang Auto. Region & DQ367040 & DQ845922 & AY625701* \\
\hline & Misgurnus sp & & AY804103* & $\mathrm{AB} 242171^{*}$ & $\mathrm{AB} 242171^{*}$ \\
\hline & Pseudogastromyzon fangi & Hengxian, Guangxi Zhuang Auto. Region & DQ367042 & DQ845920 & DQ105221 \\
\hline & Gyrinocheilus sp & & AY804074* & AB242164* & AB242164* \\
\hline
\end{tabular}

a) The cyprinid subfamilies follow those proposed by Chen [4]. * indicates sequences downloaded from GenBank, and - indicates missing data. 
initial denaturation step at $94^{\circ} \mathrm{C}$ for $3 \mathrm{~min}$, followed by 35 cycles of $30 \mathrm{~s}$ at $94^{\circ} \mathrm{C}$, annealing for $30 \mathrm{~s}$ at $45-56^{\circ} \mathrm{C}$ (depending on the gene amplified), extension for $90 \mathrm{~s}$ at $72^{\circ} \mathrm{C}$, and a final extension for $8 \mathrm{~min}$ at $72^{\circ} \mathrm{C}$. Amplified DNA was fractionated by electrophoresis through $0.8 \%$ lowmelting agarose gels, recovered, and purified using BioStar Glassmilk DNA purification Kit (Biostar International, Toronto, ON, Canada) according to the manufacturer's instructions. Nucleotide sequences were determined using purified PCR product. We generated most of the sequences used in this study, and some sequences for the 16S rRNA and Cytb genes were obtained from GenBank (Table 1).

For the two protein-coding genes, RAG2 and Cytb, multiple sequence alignments were performed using CLUSTAL $X$ [30]. For the 16S rRNA gene, sequences were initially aligned using CLUSTAL X, then manually aligned based on secondary structural elements and conserved motifs by comparing to existing models of $16 \mathrm{~S}$ rRNA secondary structure for cyprinid fishes [31-33]. All data-sets analyzed for this study are available on request from the first author.

\subsection{Data partitions and model selection}

We performed partitioned analyses using different nucleotide substitution models and associated parameter for each data subsets. We evaluated ten distinct partitioning strategies ranging from unpartitioned to a maximum of eight partitions (Table 2). Each partitioning strategies were denoted with the letter $\mathrm{P}$ followed by the number of data partitions. The unpartitioned (P1) analyses applied a single model of sequence evolution to all the data. The eight-partition (P8) analyses included separated substitution models for the stems and loops of 16S rRNA gene and for each codon position of Cytb and of RAG2.

Model selection was undertaken using PAUP [34] and ModelTest 3.7 [35]. The Akaike information criterion (AIC) weighting [36] determined the best-fit nucleotide model for each data partition. The initial tree used in ModelTest was drawn arbitrarily from a set of equally-parsimonious trees obtained with the complete data [23]. Because MrBayes 3.1.2 [21] only allows models with one, two, or six substitu- tion rates, the AIC-selected model was often impossible to implement. Consequently we were forced to choose between under- and over-parameterized models. In these situations, a feasible solution is to select the best over-parameterized model to avoid the possible negative consequences of under-parameterization, e.g., underestimated branch lengths and consequent long-branch attraction [23]. This recommendation has been verified by several simulation studies that found few costs associated with model over-parameterization, at least within the framework of the general time-reversible (GTR) family of models [37,38]. The model $\mathrm{GTR}+\mathrm{I}+\Gamma$ was applied to all partitions in our Bayesian and ML phylogenetic analyses.

\subsection{Phylogenetic analyses of the Cyprinidae}

Bayesian phylogenetic analyses were performed using the software MrBayes 3.1.2 [21]. A Metropolis-coupled Markov chain Monte Carlo (MCMC) process was undertaken for each data partition running simultaneously with a cold chain and three incrementally heated chains. The default setting for the heating parameter $(T=0.2)$ in our preliminary analyses resulted in no or infrequent state exchanges between chains. When an alternative temperature regime $(T=0.02)$ was used, successful state exchanges between chains improved in proportion to $40 \%-80 \%$.

MCMC analyses of each data partition were run for $2 \times 10^{7}$ generations, with sampling every 1000 generations. We employed two strategies to confirm stationarity. First, we plotted log-likelihood scores, tree lengths, and all model parameter values against generation number using Tracer v. 1.4 (http://tree.bio.ed.ac.uk/software/tracer/) to graphically evaluate "burn-in". Second, MCMC convergence was assessed graphically using the cumulative function of AWTY [39]. The cumulative function was used to analyze the posterior probability $(P P)$ support values for each clade to verify that these values were stable across all post-burn-in generations within each analysis. The PPs should stabilize once the Markov chain reaches stationarity, and substantial deviation of PPs from equilibrium values over time would indi-

Table 2 Partitioning strategies used in the Bayesian and maximum likelihood (ML) phylogenetic analyses, with their HMLi and - $\ln L$ scores

\begin{tabular}{|c|c|c|c|}
\hline Partition strategy & Partition identity & HMLi (Bayesian) & $-\ln L(\mathrm{ML})$ \\
\hline $\mathrm{P} 1$ & All data combined & 73918.6 & 73819.135 \\
\hline $\mathrm{P} 2$ & mtDNA; RAG2 & 73252.4 & 73241.452 \\
\hline P3 & 16S; Cytb; RAG2 & 72874.8 & 72985.832 \\
\hline $\mathrm{P} 4$ & 16S stems; 16S loops; Cytb; RAG2 & 72510.5 & 72648.3 \\
\hline P5a & 16S; Cytbpos1; Cytbpos2; Cytbpos3; RAG2 & 72177.9 & 72061.933 \\
\hline P5b & 16S; Cytb; RAG2pos 1; RAG2pos2; RAG2pos3 & 72496.1 & 72628.226 \\
\hline P6b & 16S stems; 16S loops; Cytb; RAG2pos1; RAG2pos2; RAG2pos3 & 72130.61 & 72291.174 \\
\hline P7 & 16S; Cytbpos1; Cytbpos2; Cytbpos3; RAG2pos1; RAG2pos2; RAG2pos3 & 71790.49 & 71704.833 \\
\hline P8 & 16S stems; 16S loops; Cytbpos1; Cytbpos2; Cytbpos3; RAG2pos1; RAG2pos2; RAG2pos3 & 71419.06 & 71360.693 \\
\hline
\end{tabular}


cate a lack of chain convergence. Our diagnoses suggested that chain convergence generally occurred within the first two million generations of each analysis. We followed a conservative approach by discarding the first 10 million generations as burn-in and using the remaining 10 million generations (10000 sampled trees) in all subsequent analyses. The $50 \%$ majority-rule consensus trees were generated with mean branch-length estimates, $P P$ values for each node, credible sets of trees, and parameter estimates.

Trees resulting from our partitioned analyses that explicitly accommodated among-partition rate variation (APRV) had greater harmonic mean log likelihoods (HMLi) than those from equivalent analyses that did not accommodate APRV. Therefore, we employed the "prset ratepr=variable" option in MrBayes in all partitioned analyses. In all MCMC runs, we assigned uniform priors to trees and parameters of models of sequence evolution, and an exponential prior to branch lengths.

Partitioned and unpartitioned ML analyses were performed in RAxML [22]. Following the recommendation of McGuire et al. [23], we performed two sets of analyses for each partitioning strategy. In the first set of analyses, we estimated the ML values, which were used in the ML strategy-selection procedure, of the P8 Bayesian topology under each partitioning strategy. In the second set of analyses, we searched for the ML topology with the highest likelihood during 200 runs on distinct starting trees, then used 500 bootstrap replicates to measure support for the recovered clades. We employed the GTRGAMMAI substitution model in both sets of analyses.

\subsection{Evaluation of alternative partitioning strategies}

Alternative partitioning strategies were evaluated using four different criteria [23]: standard Bayes factors [40,41], a modified AICc [23], the Bayes information criterion (BIC) [42], and a decision-theoretic (DT) approach [43-45]. The Bayes factor for any pair of partitioned models was the ratio of their marginal likelihoods. Marginal likelihoods are difficult to calculate, but can be approximated by the HMLi [46]. Using ln-transformed Bayes factors, we accepted Bayes factors greater than 10 (2ln Bayes factors $>10)$ as strong support for the more partitioned model. Because the relationships of HMLi's under alternative partitioning strategies are similar to the relationships of ML values $[23,47,48]$, we substituted the HMLis for ML values in the AICc, BIC, and DT tests of partition strategies under Bayesian framework. The partitioning strategy preferred by AICc, BIC, or DT had the minimum observed value. To estimate branch-length on a fixed-topology in MrBayes, the program's branch-swapping functionality was disabled and node-slider was enabled (by resetting props).

We compared the optimal partitioning strategies selected by Bayes factors, AICc, BIC, and DT tests in Bayesian analyses with those preferred by hierarchical LRT (hLRT),
AICc, BIC, and DT in ML analyses. To better compare ML and Bayesian strategy-selection procedures, RAxML and Bayesian analyses employed only the $\mathrm{GTR}+\mathrm{I}+\Gamma$ substitution model. All ML comparisons were based on likelihoods calculated for the eight-partition Bayesian consensus tree, whereas Bayesian model criteria were computed in the context of optimized trees for each partitioning strategy (except DT). To apply these partition selection criteria to our ML and Bayesian analyses, we calculated the number of parameters in each data partition following the recommendation of McGuire et al. [23].

\subsection{Testing alternative cyprinid phylogenetic hypo- theses}

Bayesian hypothesis testing [49] was used to test whether alternative hypotheses of higher-level cyprinid relationships recovered in our partitioned Bayesian analyses could be rejected by the combined data. Because Bayesian analysis infers the distribution of trees proportional to their PPs, commonly used statistical methods to compare alternative topologies, such as the approximately unbiased test [50], are not plausible under the Bayesian framework. The $95 \%$ credible sets of tree (sampled at stationarity) was built by using the "sumt" command in MrBayes. All trees were imported into PAUP and filtered by the phylogenetic hypothesis of interest; that hypothesis could not be rejected statistically when one or more trees in the $95 \%$ credible set compatible with the hypothesis.

\subsection{Molecular dating of cyprinids}

Rate heterogeneity among lineages in the concatenated dataset was evaluated using LRTs comparing log likelihoods of both constrained and unconstrained trees. We used the $\mathrm{GTR}+\mathrm{I}+\Gamma$ substitution model. A strict molecular clock was rejected $(P<0.005$, degrees of freedom=107). Therefore, the relaxed molecular clock model of Sanderson's nonparametric rate smoothing (NPRS) method [51] was used to estimate divergence dates.

The NPRS implemented in the program r8s [52] was used to produce ultrametric trees. Divergence date estimates were based on the topology resulting from the unpartitioned Bayesian analysis. Powell's algorithm for optimizing the objective function and the additive penalty function were used. The $95 \%$ confidence intervals for the estimated ages were determined using 100 bootstrap pseudoreplicates of the combined data matrix using SEQBOOT in PHYLIP 3.5c [53]. While keeping the tree fixed, nodal depth (hence age estimates) of each pseudoreplicate was estimated by ML with the preferred model of molecular evolution [51]. For each node, the mean age was calculated from 100 bootstrap ages.

To estimate divergence times, we applied multiple fossil calibration points including (i) the root node of Cyprinidae 
was constrained to a maximum of 55.8 million years ago (Mya) because the oldest reliable known fossils of Cyprinidae are from the Eocene [54]; (ii) the split between Tinca and the modern leuciscins was constrained to be a maximum of 18.0 Mya, because Tinca was described from the Middle Miocene [55,56] and a prominent turnover of European freshwater fish faunas represented by the appearance of modern Palaeoleucisus sp. and Palaeocarassius sp. (=aff. Abramis sp. vel aff. Alburnus sp.) happened about 17-18 Mya (the late early Miocene) [57]; (iii) a minimum of 1.81 Mya was assigned to the node subtending silver ( $H y$ pophthalmichthys molitrix) and bighead (Aristichthys nobilis) carp, and to the node subtending grass carp (Ctenopharyngodon idella) [58]; (iv) a minimum age of 3 Mya was used to define the origin of Pseudorasbora [58]; (v) a fixed date of 13 Mya was used to define the lineage leading to modern European Barbus barbus according to the fossil records of Barbus [10].

\section{Results}

We generated 4257 aligned base pairs (bp) of DNA sequence data representing three genes, the nuclear RAG2 1287 aligned bp and the 16S rRNA $1830 \mathrm{bp}$ and Cytb 1140 bp. Of those sites, 2209 were variable and 1797 were parsimony informative. In the $16 \mathrm{~S}$ rRNA gene, 190 sites were variable and 106 parsimony informative. The Cytb gene had 160 variable and 140 parsimony informative sites. The remaining 290 variable sites, 270 of which were parsimony informative, occurred in the RAG2 gene.

\subsection{Effects of alternative partitioning strategies}

The HMLi and $-\ln L$ were used to evaluate partitioning strategies in the Bayesian and ML analyses, respectively. In the present study, adding partitions substantially improved the HMLi and $-\ln L$ scores (Table 2), suggesting that simpler partitioning strategies were poorer fits to the data than more complicated partitioning strategies. For example, partitioning Cytb and RAG2 by codon positions dramatically improved HMLi and $-\ln L$. Partitioning the $16 \mathrm{~S}$ rRNA gene by stems and loops improved HMLi and $-\ln L$ by about 360 and 330 log-units respectively (P4 vs. P3). Comparing the strategies with the same numbers of partitions (P5a vs. P5b, P6a vs. P6b) indicated that partitioning Cytb alone by codon was better than partitioning only RAG2 by codon. The P8 strategy, which partitioned the rRNA gene by stems and loops and the coding genes by codon position proved best in both Bayesian and ML analyses.

Despite differences in model fit, tree topologies inferred by Bayesian and ML methods using the ten partition strategies were almost identical to one other; the differences involved alternative placements of weakly supported nodes $(P P<0.90$ and bootstrap support $<70 \%)$. The most dramatic difference in topology occurred in the position of Danio within Cyprinidae; the Bayesian P7 analysis weakly supported a basal position for Danio, unlike the other analyses. Tree length estimates varied only slightly across partitioning strategies, and no notable differences in PPs (Bayesian analyses) or bootstrap supports (ML analyses) were found among strategies. The number of strongly-supported ingroup nodes $(P P$ values $\geqslant 0.95)$ decreased between the unpartitioned (73 of 95 ingroup nodes with $P P$ values $\geqslant 0.95$ ) and the maximally partitioned (63 of 93). Our analyses suggested that highly-partitioned Bayesian analyses had relatively poor performance in recovering well-supported cyprinid nodes.

\subsection{Selecting the optimal partitioning strategy}

Two extreme partitioning strategies were selected by DT and Bayes factors (hLRT), AICc, and BIC. The DT selected the unpartitioned P1 strategy; in contrast, the other criteria preferred the most partitioned (P8) strategy. The morepartitioned strategies considered in this study did not provide better-resolved or -supported estimates of cyprinid phylogeny, because all strategies resulted in similar topologies and node support values. The partitioning strategy employed in this analysis was not as critical as expected. However, the phylogenetic analysis based on P1 (preferred by DT in both Bayesian and ML frameworks) required fewer parameters to be estimated, and we inferred that P1 strategy was optimal for our Bayesian and ML analyses. Although adding partitions improved likelihood scores, partitioning had little effect on topology or node support. Much of the improvement in likelihood scores obtained with more extensive partitioning was probably associated with substitution model and base frequency parameter estimates (nuisance parameters in this context) rather than with more critical topology and branch-length estimates [23].

\subsection{Phylogeny of the Cyprinidae}

Bayesian analyses of a combined molecular dataset resulted in informative phylogenetic estimates for cyprinids (Figure 1). The monophyly of Cyprinidae was strongly supported with a $P P$ of 1.0 in all analyses. The unpartitioned Bayesian analysis resulted in a well-resolved and -supported cyprinid phylogeny, with 73 of 95 ingroup nodes receiving $P P$ values $\geqslant 0.95$ and two additional nodes with $P P$ values of $0.90-0.95$.

The unpartitioned Bayesian analysis strongly supported several important clades within Cyprinidae. However, the position of Danio at the base of the leuciscines was poorly supported $(P P=0.61)$, and the genus was basal to the entire family in the P7 analyses. Within the cyprinine lineage (Clade I) (sensu Howes [2]), the monophyly of labeonine fishes (Clade B) and sister relationship between labeonine and non-labeonine cyprinine clades (except Procypris, Clade A) were both strongly supported $(P P=1.0)$, whereas the relationships within non-labeonine cyprinine clade 


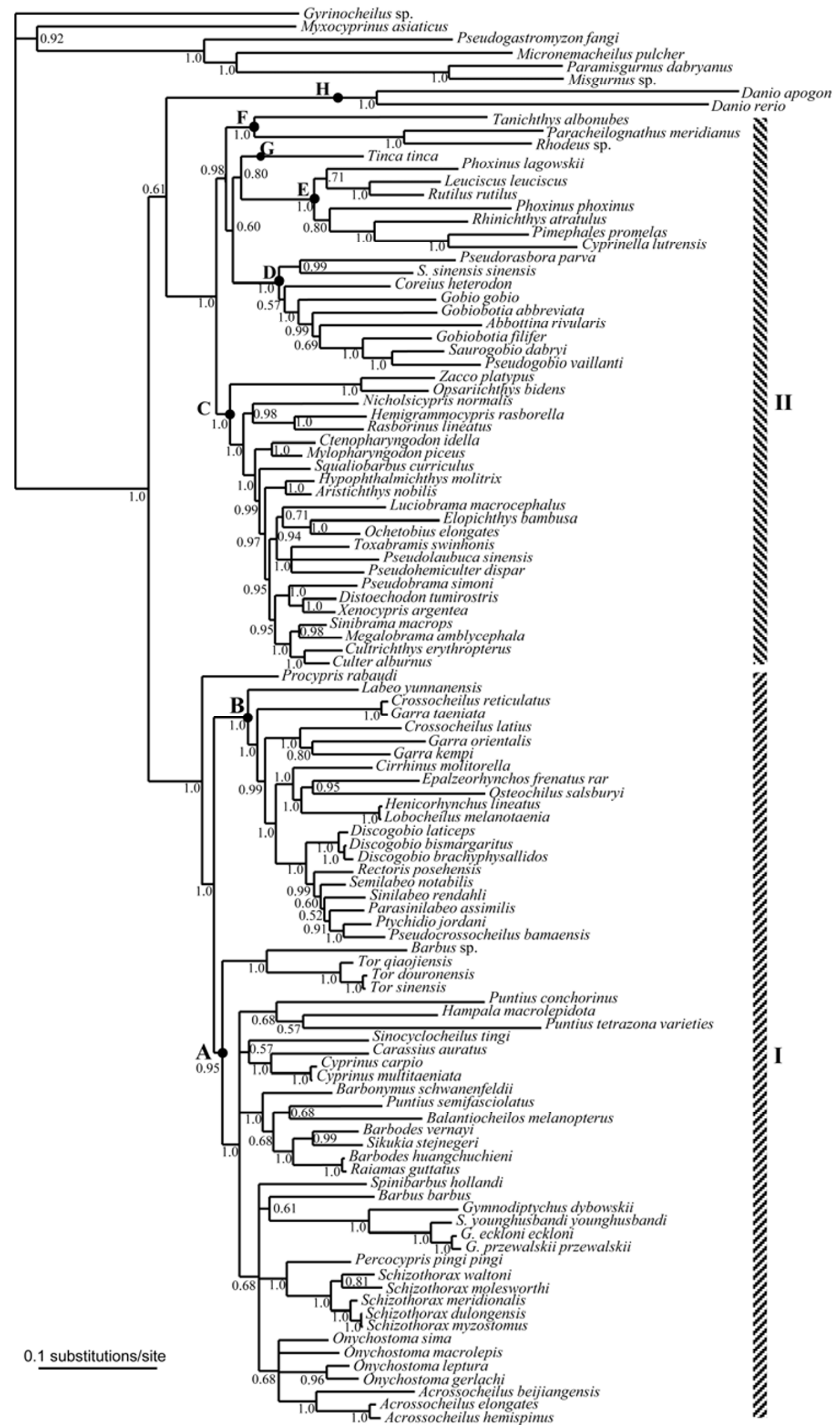

Figure 1 Phylogenetic tree of cyprinid fishes resulting from unpartitioned (P1) Bayesian analysis of three genes. Posterior probabilities values are shown at each node. Two recognized lineages within the family Cyprinidae are indicated by Roman numerals on the right side of the figure: (I) cyprinine lineage; (II) leuciscine lineage. Nodes for the recognized clades are marked with black dots and bold capital letters: A, cyprinine clade; B, labeonine clade; C, xenocyprinine clade; D, gobionine clade; E, leuciscine clade; F, acheilognathine (including Tanichthys) clade, G, tincine clade; H, danionine clade. 
(Clade A) were less well supported, with several unresolved relationships.

Within the leuciscine lineage (Clade II), the cutrins were not supported as monophyletic. However, the East Asian endemic xenocyprinine taxa (including Hypophthalmichthys, Aristichthys, Ctenopharyngodon, Mylopharyngodon, Ochetobius, Squaliobarbus, Elopichthys, Luciobrama, Culter, Cultrichthys, Sinibrama, Megalobrama, Pseudohemiculter, Toxabramis, Pseudolaubuca, Distoechodon, Xenocypris, and Pseudobrama), formed a strongly-supported clade (Clade C, $P P=1.0$ ) in which the genera Nicholsicypris, Rasborinus, Hemigrammocypris, Zacco, and Opsariichthys were well resolved as the basal members. The North American and Eurasian leuciscins formed a strongly supported clade (Clade E, $P P=1.0$ ), but its sister relationship to the genus Tinca was weakly supported $(P P=0.80)$. Clade D $(P P=1.0)$, containing gobionins and gobiobotins, was weakly supported $(P P=0.60)$ as sister to clade E+Tinca. Clade F, containing Tanichthys, Rhodeus, and Paraacheilognathus, was well supported $(P P=1.0)$, as was the sister relationship between Tanichthys and the Rhodeus + Paraacheilognathus clade $(P P=1.0)$. Furthermore, clade $\mathrm{D}, \mathrm{E}$, and $\mathrm{F}$ and Tinca formed a strongly supported clade $(P P=0.98)$ sister to the strongly-supported East Asian endemic xenocyprinine clade (Clade $C)(P P=1.0)$. The other nine partitioning strategies yielded Bayesian topologies largely in agreement with this tree.

The unpartitioned ML analysis (the strategy preferred by DT in an ML framework) and the more complex partitioning strategies resulted in phylogenetic trees highly similar to Figure 1, with the following exceptions: (i) the phylogenetic position of Danio; (ii) the deep branching pattern within Clade A; and (iii) support for the node subtending clade D, E, and F and Tinca. All of the important cyprinid clades recovered in Figure 1 were also well supported (bootstrap $\geqslant 70 \%$ ) in the ML analysis, except that the strongly-supported $(P P=0.95)$ node for Clade $\mathrm{A}$ in the Bayesian analysis had relatively low bootstrap support $(66 \%)$ in the ML analysis.

\subsection{Divergence dates of cyprinid clades}

Table 3 lists the divergence times (with 95\% confidence intervals) estimated in $\mathrm{r} 8 \mathrm{~s}$ for the main nodes marked in Figure 2. As estimated by our combined data, Cyprinidae appeared in East Asia around 42.38 (43.13-41.64) Mya. The cyprinine and leuciscine lineages separated an estimate of 27.36 (27.84-26.89) Mya. Our dating results suggested that the cyprinine and leuciscine lineages began to diversify simultaneously (CYN node, 20.45 Mya; LEU node, 20.51 Mya). Within the cyprinine lineage, radiation of Labeoninae (node LAB) occurred 14.98 Mya, while the Cyprininae (node CYT) diverged earlier (18.46 Mya). Radiation of the clades Acheilognathinae (node ACH), Gobioninae (node GOB), Leuciscinae (node LES), and Xenocyprinine (node XEN) occurred from 18.80-12.02 Mya.

\section{Discussion}

\subsection{Performance of alternative partitioning strategies}

For the datasets composed of multiple genes and/or gene regions, partitioned phylogenetic analyses may greatly reduce mismodeling and systematic errors relative to analyses specifying a single model. Comparison of the $95 \%$ credible intervals of parameters sampled from the posterior distributions of the P1 and P8 analyses found significant heterogeneity, indicating that including more partitions greatly improved the Bayesian and ML likelihoods in this study. Numerous instances of non-overlap could be found in the credible intervals for different partitioning schemes. Based on the parameter estimates, partitioning the Cytb codon positions improved the HMLi and $-\ln L$ scores more substantially than partitioning the RAG2 or 16S rRNA genes.

Although partitioning substantially improved likelihood scores, its effect on topology and node support was minimal. In our Bayesian analyses, increased partitioning decreased the estimated PPs of some nodes relative to the P1 strategy. For example, seven of the ingroup nodes that had $P P$ values

Table 3 Divergence time estimates and their 95\% confidence interval for key nodes in the cyprinid phylogeny (Figure 2)

\begin{tabular}{|c|c|c|c|}
\hline \multirow{2}{*}{ Clade } & \multirow{2}{*}{ Node } & \multicolumn{2}{|c|}{ Age estimates (Mya) } \\
\hline & & Mean \pm SD & $95 \%$ interval \\
\hline Cyprinidae, except outgroup & CYD & $42.38 \pm 3.74$ & $41.64-43.13$ \\
\hline Cyprinine lineage & CYN & $20.45 \pm 1.41$ & $20.17-20.73$ \\
\hline Cyprinine clade & CYT & $18.46 \pm 1.04$ & $18.25-18.67$ \\
\hline Labeonine clade & LAB & $14.98 \pm 0.95$ & $14.79-15.17$ \\
\hline Leuciscine lineage & LEU & $20.51 \pm 1.22$ & $20.27-20.76$ \\
\hline Leuciscine clade & LES & $12.02 \pm 0.63$ & $11.90-12.15$ \\
\hline Gobionine clade & GOB & $14.13 \pm 0.88$ & $13.95-14.30$ \\
\hline Acheilognathine clade & $\mathrm{ACH}$ & $17.11 \pm 0.97$ & $16.92-17.30$ \\
\hline Leuciscine clade and the genus Tinca & TIN & $17.90 \pm 0.40$ & $17.82-17.98$ \\
\hline Xenocyprinine clade (East Asian endemic clade) & XEN & $18.80 \pm 1.12$ & $18.58-19.02$ \\
\hline
\end{tabular}




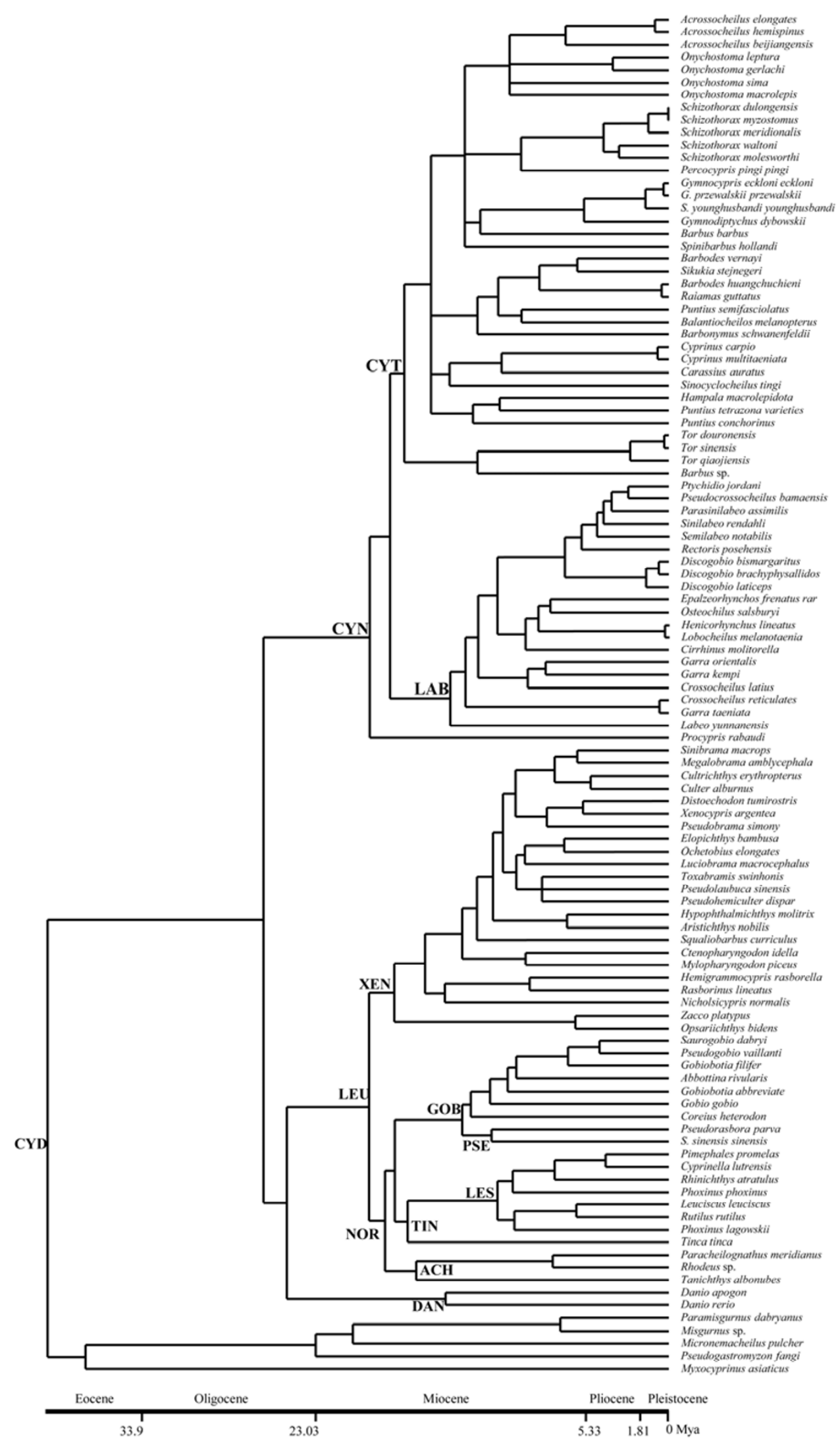

Figure 2 Phylogeny of cyprinid fishes with divergence time estimates. The chronogram is the tree from the unpartitioned Bayesian analysis with dates estimated using nonparametric rate smoothing in the program R8s. Node labels are defined in Table 3, where mean divergence dates and $95 \%$ confidence intervals for key nodes are listed. 
of 1.0 in the P1 tree had lower support values under the most partitioned (P8) strategy. Phylogeneticists are concerned about appropriate partitioning in their analyses, because poor topology and confidence estimates can result from poorly- or overly-partitioned models. Although improved modeling could decrease the amount of systematic error under a given partitioning strategy, random error could significantly impact phylogeny and confidence estimates. The ideal partition size for optimal phylogenetic estimates is still unclear. For our cyprinid dataset, we concluded that most of the improvement in HMLi and $-\ln L$ estimates with greater partitioning was associated with better estimation of nuisance parameters, such as base frequencies and substitution rates.

We compared ten partitioning strategies in both Bayesian and ML frameworks, and four alternative model-selection criteria were employed to screen the best-fitting strategy. The standard Bayes factor/hLRT and AICc imposed relatively weak penalties for additional parameterization and consequently selected the most complex partitioning strategy, whereas the more stringent BIC and DT criteria preferred the most- and least-partitioned models, respectively. The DT method incorporates relative branch-length error as a performance measure. Therefore, under the DT framework, if a less-partitioned model returned nearly identical branch length estimates to those of a model with more partitions, there would be little difference in phylogenetic estimates between the models. The performance-based DT criterion selected the unpartitioned strategy in our analyses, indicating that there were probably no improvements in branch length estimates in our partitioned (P2-P8, Table 2) analyses compared with unpartitioned analyses.

\subsection{Phylogenetic framework for systematics of the $\mathrm{Cy}$ - prinidae}

As expected, the monophyly of the family Cyprinidae was recovered with strong Bayesian PP and ML bootstrap support. Our phylogeny established a higher-level framework for Cyprinidae and revealed several well-supported groupings.

One large clade within Cyprinidae was the wellsupported cyprinine lineage (Clade I, Figure 1). All taxa in this clade were members of the previously-recognized subfamilies, Barbinae, Cyprininae, Labeoninae, and Schizothoracinae [4]. Although the basal relationships within this clade have been contentious due to disagreement between molecular and morphological phylogenetic studies, our data consistently supported the monophyly of the cyprinine clade. Within the cyprinines, our analyses provided robust evidence for the monophyly of Labeoninae as the currently recognized. However, in all of our analyses, the cyprinine, barbine, and schizothoracine fishes (except Procypris) were nested within one clade (Clade A) sister to the labeonine clade. In another analysis with more cyprinine samples (unpublished data), two clades were strongly recovered: the Labeoninae and the Cyprininae, containing the barbins, cyprinins (including Procypris), and schizothoracins.

Another well-supported primary clade of Cyprinidae resolved in all analyses was the leuciscine lineage (Clade II, Figure 1). Within this clade, all of our analyses provided substantial resolution and support for the monophyly of Gobioninae (including Gobiobotia), Acheilognathinae, Leuciscinae, and Xenocyprininae, the latter is endemic to East Asia. Although Gobioninae, Acheilognathinae, and Leuciscinae were each strongly supported, the relationships among them were weakly resolved and differed among analyses. These three clades, together with Tinca, formed a clade sister to the Xenocyprininae. The placement of Tinca within Cyprinidae has proved to be taxonomically problematic in previous studies [2]. In contrast to studies based on morphological [8,9] and molecular [10-12,20] data, our analyses strongly supported a clade comprised of Tinca, leuciscini, Gobionini, and Acheilognathini, within which Tinca was weakly supported as sister to leuciscini.

Not surprisingly, the monophyly of the danionine (rasborine sensu Howes [2]) fishes was rejected by the present analyses. Morphologically, "Danioninae" contains a large assemblage of taxa, most of which cannot be accommodated by other subfamilies [2]. Furthermore, a recent molecular phylogeny indicated that Danioninae was not monophyletic; putative members were scattered throughout Cyprinidae [59]. Thus, we suggest that the East Asia endemics, such as Zacco, Opsariichthys, and Nicholsicypris should be excluded from a redefined Danioninae .

In the recent taxonomic revision of cyprinid (or cyprinoid) fishes by Chen and Mayden [60], the recognition of 10 families (including the Psilorhynchidae) was recommended. Of these groups, six (i.e., Acheilognathinae, Leuciscinae, Gobioninae, Cultrinae, Tincinae, and Rasborinae) were also supported in the present analyses (Figure 1). The Psilorhynchidae and Leptobarbidae were not included in our analyses, and the Cultrinae and Rasborinae referred to Xenocyprininae and Danioninae, respectively, in our study. Our data suggested that the Cyprinae recognized by Chen and Mayden [60] could be further divided into two clades, Cyprininae and Labeonine, and that the Tanichthyidae should be included in the Acheilognathinae. Unlike Chen and Mayden, we do not recommend that these groups be elevated from subfamily to family level, but prefer to retain these clades within Cyprinidae.

Previous morphological studies consistently supported two major lineages within Cyprinidae, i.e., barbeled cyprinines and (usually) non-barbeled leuciscines, although the subgroups included in each lineage and the relationships among subgroups have differed among studies [2,8,9]. However, recent molecular studies have disagreed with the morphological placement of Danionine (Rasborinae). The placement of Danioninae to the leuciscine clade was indicated in some prior morphological and molecular phylogeny 
$[8,9,60]$, and was weakly supported in our cyprinid phylogeny (Figure 1). Another study placed Danioninae within the cyprinine [2], while other molecular phylogenetic analyses placed it at the base of the cyprinids [12,13,61]. The disputed phylogenetic placement of Danioninae may be mainly due to different taxon sampling in these previous molecular phylogenies. Our data indicated that Danioninae represents a lineage within Cyprinidae, that is distinct from the well-accepted cyprinine and leuciscine lineages. A basal position for Danioninae within Cyprinidae (as recovered in the P7 Bayesian analysis) could not be rejected by Bayesian hypothesis testing of alternatives phylogenies generated from our combined data. A total of 6414 of 18710 trees in the $95 \%$ credible set were congruent with the hypothesis that Danioninae was basal within cyprinids.

\subsection{Phylogenetic history of cyprinid clades}

Based on the distribution of fossil cyprinids, an Eocene origin for cyprinids was proposed [54]. Consistent with this hypothesis, our molecular dating analyses also indicated that cyprinids originated in the Middle Eocene (around 42 Mya). Within the family, the cyprinine linage appeared in the early Late Oligocene (around 27 Mya) and the leuciscine lineage in the Late Oligocene (about 26-25 Mya).

The radiation of Labeoninae, the major cyprinine clade, occurred in the early Middle Miocene, and Cyprininae was estimated to have diversified in the late Early Miocene. Within the leuciscine lineage, the divergence between Xenocyprininae and the lineage comprising Leuciscinae, Tincinae, Gobioninae, and Acheilognathinae, occurred in the Early Miocene (about 20 Mya). According to our age estimates, the Acheilognathini, Gobionini, and Leuciscini radiated during the Middle Miocene (around 18-12 Mya).

We thank Dr. Wang JiangXin for his assistance in improving the presentation of our manuscript. This work was supported by the National Natural Science Foundation of China (Grant No. 30770300) and Chinese Academy of Sciences (Grant No. KSCX2-EW-Q-12), and the Cypriniformes Tree of Life Initiative supported by the USA National Science Foundation (Grant No. EF-0431326).

1 Nelson J S, ed. Fishes of the World. New York: John Wiley and Sons Inc., 2006

2 Howes G J. Systematics and biogeography: an overview. In: Winfield I J,Nelson J S, eds. Cyprinid Fishes: Systematics, Biology and Exploitation. London: Chapman and Hall, 1991. 1-33

3 Hensel K. Review of the classification and of the opinions on the evolution of Cyprinoidei (Eventognathi) with an annotated list of genera and subgenera described since 1921. Annot Zool Bot, 1970, 57: $1-45$

4 Chen Y Y, ed. Fauna Sinica, Osteichthys: Cypriniformes (Part II). Beijing: Science Press, 1998

5 Gosline W A. Unbranched dorsal-fin rays and subfamily classification in the fish family Cyprinidae. Occas Pap Mus Zool Univ Mich, 1978, 684: 1-21

6 Chu Y T. Comparative studies on the scales and on the pharyngeals and their teeth in Chinese Cyprinids, with particular reference to taxonomy and evolution. Biol Bull St John's Univ (Shanghai), 1935, 2: $1-225$

7 Wu X, ed. The Cyprinid Fishes of China (in Chinese). Shanghai: Shanghai Science and Technology Press, 1964

8 Chen X L, Yue P Q, Lin R D. Major groups within the family Cyprinidae and their phylogenetic relationships. Acta Zootaxon Sin, 1984, 9: 424-440

9 Cavender T M, Coburn M M. Phylogenetic relationships of North American Cyprinidae. In: Mayden R L, ed. Systematics, Historical Ecology and North American Freshwater Fishes. Stanford, California: Stanford University Press, 1992. 293-327

10 Zardoya R, Doadrio I. Molecular evidence on the evolutionary and biogeographical patterns of European Cyprinids. J Mol Evol, 1999, 49: 227-237

11 Briolay J, Galtier N, Brito R M, et al. Molecular phylogeny of Cyprinidae inferred from cytochrome b DNA sequences. Mol Phylogenet Evol, 1998, 9: 100-108

12 Gilles A, Lecointre G, Faure E, et al. Mitochondrial phylogeny of the European Cyprinids: implications for their systematics, reticulate evolution, and colonization time. Mol Phylogenet Evol, 1998, 10: 132-143

13 Gilles A, Lecointre G, Miquelis A, et al. Partial combination applied to phylogeny of European Cyprinids using the mitochondrial control region. Mol Phylogenet Evol, 2001, 19: 22-33

14 Zardoya R, Doadrio I. Phylogenetic relationships of Iberian Cyprinids: systematic and biogeographical implications. Proc R Soc B Biol Sci, 1998, 265: 1365-1372

15 Durand J D, Tsigenopoulos C S, Unlu E, et al. Phylogeny and biogeography of the family Cyprinidae in the Middle East inferred from cytochrome b DNA- evolutionary significance of this region. Mol Phylogenet Evol, 2002, 22: 91-100

16 Hanfling B, Brandl R. Phylogenetics of European Cyprinids: insights from allozymes. J Fish Biol, 2000, 57: 265-276

17 Fu C Z, Wu J H, Chen J K, et al. Freshwater fish biodiversity in the Yangtze River Basin of China: patterns, threats and conservation. Biodivers Conserv, 2003, 12: 1649-1685

18 Cunha C, Mesquita N, Dowling T E, et al. Phylogenetic relationships of Eurasian and American Cyprinids using cytochrome b sequences. J Fish Biol, 2002, 61: 929-944

19 He S, Liu H, Chen Y, et al. Molecular phylogenetic relationships of Eastern Asian Cyprinidae (Pisces: Cypriniformes) inferred from cytochrome b sequences. Sci China Ser C-Life Sci, 2004, 47: 130-138

20 Liu H, Chen Y. Phylogeny of the East Asian Cyprinids inferred from sequences of the mitochondrial DNA control region. Can J Zool, 2003, 81: 1938-1946

21 Ronquist F, Huelsenbeck J P. Mrbayes 3: Bayesian phylogenetic inference under mixed models. Bioinformatics, 2003, 19: 1572-1574

22 Stamatakis A. Raxml-Vi-Hpc: maximum likelihood-based phylogenetic analyses with thousands of taxa and mixed models. Bioinformatics, 2006, 22: 2688-2690

23 McGuire J A, Witt C C, Altshuler D L, et al. Phylogenetic systematics and biogeography of hummingbirds: Bayesian and maximum likelihood analyses of partitioned data and selection of an appropriate partitioning strategy. Syst Biol, 2007, 56: 837-856

24 Fink S V, Fink W L. Interrelationships of the ostariophysan fishes (Teleostei). Zool J Linn Soc, 1981, 72: 297-353

25 Wang X, Wang J, He S, et al. The complete mitochondrial genome of the Chinese hook snout carp Opsariichthys bidens (Actinopterygii: Cypriniformes) and an alternative pattern of mitogenomic evolution in vertebrate. Gene, 2007, 399: 11-19

26 Sambrook J, Fritsch E, Maniatis T, ed. Molecular Cloning: A Laboratory Manual. New York: Cold Spring Harbor Laboratory Press, 1989

27 Xiao W H, Zhang Y P, Liu H Z. Molecular systematics of Xenocyprinae (Teleostei : Cyprinidae): taxonomy, biogeography, and coevolution of a special group restricted in East Asia. Mol Phylogenet Evol, 2001, 18: 163-173

28 Li J, Wang X, Kong X, et al. Variation patterns of the mitochondrial 
16s rRNA gene with secondary structure constraints and their application to phylogeny of Cyprinine fishes (Teleostei: Cypriniformes). Mol Phylogenet Evol, 2008, 47: 472-487

29 Lovejoy N R, Collette B B. Phylogenetic relationships of new world needlefishes (Teleostei: Belonidae) and the biogeography of transitions between marine and freshwater habitats. Copeia, 2001, 2: 324-338

30 Thompson J D, Gibson T J, Plewniak F, et al. The Clustal_X Windows interface: flexible strategies for multiple sequence alignment aided by quality analysis tools. Nucleic Acids Res, 1997, 25: 4876-4882

31 De Rijk P, Wuyts J, Van de Peer Y, et al. The European large subunit ribosomal RNA database. Nucleic Acids Res, 2000, 28: 177-178

32 Gutell R R, Gray M W, Schnare M N. A compilation of large subunit (23s and 23s-like) ribosomal RNA structures. Nucleic Acids Res, 1993, 21: 3055-3074

33 Gutell R R, Fox G E. A compilation of large subunit RNA sequences presented in a structural format. Nucleic Acids Res, 1988, 16: r175-269

34 Swofford D L. Paup*: Phylogenetic Analysis Using Parsimony (*and Other Methods), Version 4.0b10. 2003

35 Posada D, Crandall K A. Modeltest: testing the model of DNA substitution. Bioinformatics, 1998, 14: 817-818

36 Posada D, Buckley T R. Model selection and model averaging in phylogenetics: advantages of Akaike information criterion and Bayesian approaches over likelihood ratio tests. Syst Biol, 2004, 53: 793-808

37 Lemmon A R, Moriarty E C. The importance of proper model assumption in Bayesian phylogenetics. Syst Biol, 2004, 53: 265-277

38 Huelsenbeck J, Rannala B. Frequentist properties of Bayesian posterior probabilities of phylogenetic trees under simple and complex substitution models. Syst Biol, 2004, 53: 904-913

39 Wilgenbusch J, Warren D, Swofford D. Awty: A System for Graphical Exploration of Mcmc Convergence in Bayesian Phylogenetic Inference. 2004

40 Kass R E, Raftery A E. Bayes factors. J Am Stat Assoc, 1995, 90: 773-795

41 Nylander J A A, Ronquist F, Huelsenbeck J P, et al. Bayesian phylogenetic analysis of combined data. Syst Biol, 2004, 53: 47-67

42 Schwarz G. Estimating the dimension of a model. Ann Stat, 1978, 6: $461-464$

43 Minin V, Abdo Z, Joyce P, et al. Performance-based selection of likelihood models for phylogeny estimation. Syst Biol, 2003, 52: 674-683

44 Abdo Z, Minin V N, Joyce P, et al. Accounting for uncertainty in the tree topology has little effect on the decision-theoretic approach to model selection in phylogeny estimation. Mol Biol Evol, 2005, 22: 691-703

45 Sullivan J, Abdo Z, Joyce P, et al. Evaluating the performance of a successive-approximations approach to parameter optimization in maximum-likelihood phylogeny estimation. Mol Biol Evol, 2005, 22: 1386-1392

46 Raftery A. Hypothesis testing and model selection. In: Gilks W R, Spiegelhalter D J, Richardson S, eds. Markov Chain Monte Carlo in Practice. London: Chapman and Hall, 1996. 163-187

47 Castoe T A, Sasa M M, Parkinson C L. Modeling nucleotide evolution at the mesoscale: the phylogeny of the neotropical pitvipers of the Porthidium group (Viperidae: Crotalinae). Mol Phylogenet Evol, 2005, 37: 881-898

48 Castoe T A, Parkinson C L. Bayesian mixed models and the phylogeny of pitvipers (Viperidae: Serpentes). Mol Phylogenet Evol, 2006, 39: 91-110

49 Brandley M C, Schmitz A, Reeder T W. Partitioned Bayesian analyses, partition choice, and the phylogenetic relationships of scincid lizards. Syst Biol, 2005, 54: 373-390

50 Shimodaira $\mathrm{H}$. An approximately unbiased test of phylogenetic tree selection. Syst Biol, 2002, 51: 492-508

51 Sanderson M J. A nonparametric approach to estimating divergence times in the absence of rate constancy. Mol Biol Evol, 1997, 14: 1218-1231

52 Sanderson M J. R8s: inferring absolute rates of molecular evolution and divergence times in the absence of a molecular clock. Bioinformatics, 2003, 19: 301-302

53 Felsenstein J. Phylip (Phylogeny Inference Package) Version 3.5 C. 1993

54 Cavender T M. The fossil record of the Cyprinidae. In: Winfield I J, Nelson J S, eds. Cyprinid Fishes: Systematics, Biology and Exploitation. London: Chapman and Hall, 1991. 34-54

55 Hierholzer E, Mörs T. Cypriniden-Schlundzähne (Osteichthyes: Teleostei) aus dem Tertiär von Hambach (Niederrheinische Bucht, Nw-Deutschland). Palaeontographica, Abteilung A, 2003, 269: 1-38

56 Sytchevskaya E. Freshwater Ichthyofauna of the Neogene of Mongolia. Tr Sovm Sovets-Mongol Paleontol Eksped, 1989, 39: $1-144$

57 Schulz-Mirbach T, Reichenbacher B. Reconstruction of oligocene and neogene freshwater fish faunas-an actualistic study on cypriniform otoliths. Acta Palaeontol Pol, 2006, 51: 283-304

58 Liu H, Su T. Pliocene fishes from the Yushe Basin, Shanxi. Vertebr Palasiat, 1962, 6: 1-25

59 Tang K L, Agnew M K, Hirt M V, et al. Systematics of the subfamily Danioninae (Teleostei: Cypriniformes: Cyprinidae). Mol Phylogenet Evol, 2010, 57: 189-214

60 Chen W J, Mayden R L. Molecular systematics of the Cyprinoidea (Teleostei: Cypriniformes), the world's largest clade of freshwater fishes: further evidence from six nuclear genes. Mol Phylogenet Evol, 2009, 52: 544-549

61 Wang X, Li J, He S. Molecular evidence for the monophyly of East Asian groups of Cyprinidae (Teleostei: Cypriniformes) derived from the nuclear recombination activating gene 2 sequences. Mol Phylogenet Evol, 2007, 42: 157-170

Open Access This article is distributed under the terms of the Creative Commons Attribution License which permits any use, distribution, and reproduction in any medium, provided the original author(s) and source are credited. 